\title{
Environmentally-Friendly RF Plasma Treatment of Thai Silk Fabrics with Chitosan for Durable Antibacterial Property
}

\author{
Doonyapong Wongsawaeng $^{1, \mathrm{a}, *}$, Sarayut Khemngern ${ }^{1, \mathrm{~b}}$, and Naraporn Somboonna ${ }^{2}$ \\ 1 Nuclear Engineering Department, Faculty of Engineering, Chulalongkorn University, Bangkok, Thailand \\ 10330 \\ 2 Department of Microbiology, Faculty of Science, Chulalongkorn University, Bangkok, Thailand 10330 \\ E-mail: aDoonyapong.W@Chula.ac.th (Corresponding author), bsarayutk@outlook.co.th
}

\begin{abstract}
A $13.56 \mathrm{MHz}$ RF plasma discharge was successfully utilized to activate and coat Thai silk fabrics with chitosan for durable antibacterial property. Uncolored and untreated Thai silk fabrics were activated in Ar plasma for 5 minutes with an optimized RF power of $120 \mathrm{~W}$ under Ar pressure of 0.8 Torr. After plasma activation, specimens were submerged and stirred in a $1 \%(\mathrm{w} / \mathrm{v})$ chitosan solution. FTIR analysis confirmed the presence of chitosan on the silk fabrics. From SEM analysis, chitosan was observed to coat silk fibers almost everywhere. RF plasma treated fabrics were able to absorb the chitosan solution substantially faster than those without the treatment. Another benefit of increased hydrophilicity is the ability of the treated fabrics to allow human perspiration to flow through more effectively, providing additional comfort when worn in tropical-zone countries. Antibacterial properties against E. coli and S. aureus reduced with increasing washing cycles dropping to approximately $95 \%$ after 5 rounds of washing. Extrapolation of linear trend lines revealed that for the case of E. coli, it will take approximately 16 washing cycles to reduce the antibacterial ability to $90 \%$. For the case of S. aureus, it will take approximately 10 washing cycles to reduce the antibacterial ability to $90 \%$. Fabrics without RF plasma treatment prior to chitosan solution submersion will have their antibacterial ability for E. coli and S. aureus reduced to $90 \%$ after only about 5 washing cycles. Thus, RF plasma treatment can effectively induce chitosan to provide a strong and durable coating for Thai silk fabrics, thus, offering a new, very environmentally-friendly coating technique for Thai silk with chitosan for potential use in the textile industry.
\end{abstract}

Keywords: $13.56 \mathrm{MHz}$ RF plasma, Thai silk, chitosan, antibacteria.

ENGINEERING JOURNAL Volume 21 Issue 1

Received 16 February 2016

Accepted 30 May 2016

Published 31 January 2017

Online at http://www.engj.org/

DOI:10.4186/ej.2017.21.1.29 


\section{Introduction}

Plasma treatment on textiles usually refers to the following three processes. The first is plasma polymerization, which is used to deposit a polymeric film on a substrate under the influence of plasma. This technique offers several advantages over conventional polymer synthesis techniques, which include ultrathin, pin-hole free films with thicknesses of $500 \AA$ to $1 \mu \mathrm{m}$ can be synthesized; films can adhere to a variety of substrates such as polymers, glasses and metals; and polymerization can be achieved without the use of solvents (vapor phase polymerization) [1]. Moreover, functional groups of monomers can be retained, and a highly cross-linked and dense coating can easily be synthesized. Synthesized films have been widely used in various applications such as anticorrosive surfaces, humidity sensors, electrical resistors, scratch resistant coatings, optical filters, protective coatings and chemical barrier coatings [2]. The second plasma treatment process is plasma grafting after an activation of the surface by plasma discharge [3]. Similar to plasma polymerization, various monomers can be grafted/graft-polymerized onto various surfaces. Functional groups of monomers can result in the surface exhibiting desired properties such as hydrophobicity, flame retardancy and antibacterial ability without affecting the bulk property of the material. The third plasma treatment process is surface modification by plasma such as surface etching, which is usually performed by noble gases.

Common sources of energy to ionize the gas in the reaction chamber are direct current (DC), radio frequency (RF, typically $13.56 \mathrm{MHz}$ ) and microwave (typically $2.45 \mathrm{GHz}$ ). Reaction chamber design, feed gas composition and flow rate, RF frequency and power, plasma pressure, substrate temperature (lower temperature, higher deposition rate) and position in the chamber all influence the film deposition rate and physical and chemical properties of the film and the grafting [1].

Thai silk fabric has long been considered a popular product of Thailand. Various properties of silk fabric can be improved for comfort, safety and hygienics such as hydrophobicity, flame retardancy and antibacterial. Plasma treatment can be used to effectively enhance these surface-related properties because the process can be used to graft an appropriate material on the silk fiber surface without affecting the overall bulk mechanical properties. For example, hydrophobicity enhancement of Thai silk using $\mathrm{SF}_{6}$ plasma has been successfully performed in an inductively coupled plasma discharged system [3, 4]. Improvement of hydrophobic properties of Chinese silk and cotton by $\mathrm{C}_{3} \mathrm{~F}_{6}$ plasma treatment has also been successfully performed [5]. A plasma induced graft polymerization was successfully utilized to coat silk fabrics with flame retardant material, followed by $\mathrm{SF}_{6}$ plasma treatment for water repellent finishing [6]. Enhancement of hydrophobicity and tensile strength of Indian muga silk fiber by RF plasma discharge has also been successfully performed [7].

To increase antibacterial ability for surface enhancement, silk fiber surface was modified using anhydrides (succinic anhydride and phthalic anhydride) in different solvents (dimethyl sulfoxide (DMSO) and N,N-dimethyl formamide (DMF)) to chemically graft chitosan [8]. Grafted fibers were expected to exhibit an antibacterial property due to the presence of chitosan on the surface. Wool fabric was also grafted with chitosan using the same chemical grafting technique [9].

Chitosan is a biopolymer produced by alkaline deacetylation of chitin, which is the most abundant biopolymer in nature after cellulose. It is commonly extracted from shrimp and crab shells. Chitosan is highly bio-compatible and has been widely used in several countries as a natural preservative, as a coating for fruits and vegetables to preserve their freshness and as edible films for containing food, for example. As chitosan can induce Trichoderma harzianum such as Chitinase enzyme or $\beta$-1, 3-glucanase enzyme, which can dissolve septate hypha of fungi and cell walls of microbial organisms, this eventually results in their deaths. Thus, chitosan has also widely been used as anti-fungi and antibacterial agents for many agricultural products [10-15].

There is no literature report on grafting chitosan onto Thai silk or any other silk using a $13.56 \mathrm{MHz} \mathrm{RF}$ plasma treatment process. Thus, this work offers a new approach to coat fabrics. At this point, a question may arise as to whether it will be possible to graft chitosan onto a surface using RF plasma discharge. Chitosan was reported to be successfully grafted onto a multiwall carbon nanotubes (MWCNTs) surface using RF plasma [16]. The grafting procedure was performed by first treating the MWCNTs in $\mathrm{N}_{2}$ plasma in a grafting reactor. The surface-activated MWCNTs were subsequently dipped in a $80^{\circ} \mathrm{C}, 1.50 \mathrm{~g} / \mathrm{L}$ chitosan solution. The active carbon sites on the carbon nanotube surface can react with chitosan. The chitosan functional groups of $-\mathrm{CH}_{2} \mathrm{OH},-\mathrm{OH}$ and $-\mathrm{NH}_{2}$ can form $\mathrm{C}-\mathrm{O}-\mathrm{C}, \mathrm{C}-\mathrm{O}-\mathrm{C}$ and $\mathrm{C}-\mathrm{NH}-\mathrm{C}$ can 
then bond between chitosan and MWCNTs [16]. Moreover, chitosan was successfully grafted onto nylon textiles by placing open-air plasma activated nylon textiles in a chitosan solution [17].

Low-temperature RF plasma treatment of silk fabrics with chitosan is advantageous over the wet chemical treatment process because of the following reasons: 1) The only required chemicals for grafting are chitosan, acetic acid and $\mathrm{NaOH}$, making this process highly environmentally-friendly compared to the chemical grafting process which requires anhydrides and solvent such as DMSO or DMF [8]; 2) Because only environmentally-friendly chemicals are used, when fabrics reach their end of life, there will be no chemical discharged to the environment; 3) Because plasma treatment is a closed system, byproducts (if any) can be trapped rather than being released to the environment; 4) The process is worker-friendly [18]; 5) Plasma treatment alters only the skin of the fabric without affecting bulk properties, while for the chemical process, chemicals used may affect bulk properties; 6) Although the initial investment for plasma equipment and high vacuum system can be high, the long-term saving from not using chemicals can eventually outweigh the initial investment.

The objective of this experimental work was to effectively and durably graft Thai silk fabrics with chitosan using the environmentally-friendly RF plasma treatment process to increase the antibacterial property. The chitosan-grafted fibers were analyzed for the presence of chitosan using scanning electron microscopy (SEM) and fourier transform infrared spectroscopy (FTIR). Antibacterial properties were evaluated by AATCC 100 protocol (conventional antibacterial diagnostic on textile materials) for Escherichia coli (E. coli) and Staphylococcus aureus (S. aureus). E. coli bacteria can cause food poisoning exhibiting symptoms such as abdominal cramps and diarrhea. S. aureus is common on human skin and although not always pathogenic, can cause skin diseases, especially atopic dermatitis. Additionally, Staphylococcus spp. including $S$. aureus can cause body smell and color on textiles. Hence, antibacterial finish not only protects human skin but also prevents undesired body odor while it prolongs textile color, and these are reasons why Thai silk needs an antimicrobial coating. Finally, to assess the durability of the chitosan coating, grafted fabrics underwent a series of laundering durability tests, and its antibacterial ability was evaluated.

\section{Experimental}

\subsection{Plasma Chamber}

The RF power supply system consisted of a $13.56 \mathrm{MHz}$ RF power generator made by DryTek, model 2600422. The output signal was connected to an automatic matching network made by YSE System, model AMN-200. The automatic matching network was required to match the impedance of the generator to that of the load to minimize reflected power back to the generator. For all the runs, the reflected power was detected to be less than 5 watts. Thus, the forward power was approximately the power generating the plasma. The gas supply system consisted of a UHP Ar gas tank connected to a Unit Instruments mass flow controller. A rotary mechanical vacuum pump was used to maintain vacuum in the chamber. A GranvillePhillips convectron vacuum gauge and a reader were employed to measure the vacuum level in the chamber. A combination of the gas flow rate and the position of the isolation valve placed between the pump and the plasma chamber established the desired vacuum level in the chamber.

The glass plasma chamber had the inner diameter of $21 \mathrm{~cm}$ and the length of $31 \mathrm{~cm}$. The substrate holder and the high potential plate placed parallel to each other were made of copper with the dimensions of $15 \mathrm{~cm} \times 22 \mathrm{~cm}$. They were fixed and separated from each other by $1 \mathrm{~cm}$ using high-purity alumina rods. A stainless steel gas supply line was inserted into the chamber and the opening of the line was between the substrate holder and the high potential plate to ensure that the argon plasma was sufficiently and uniformly generated in the substrate holder area. Figure 1 shows a diagram of the experimental setup. 


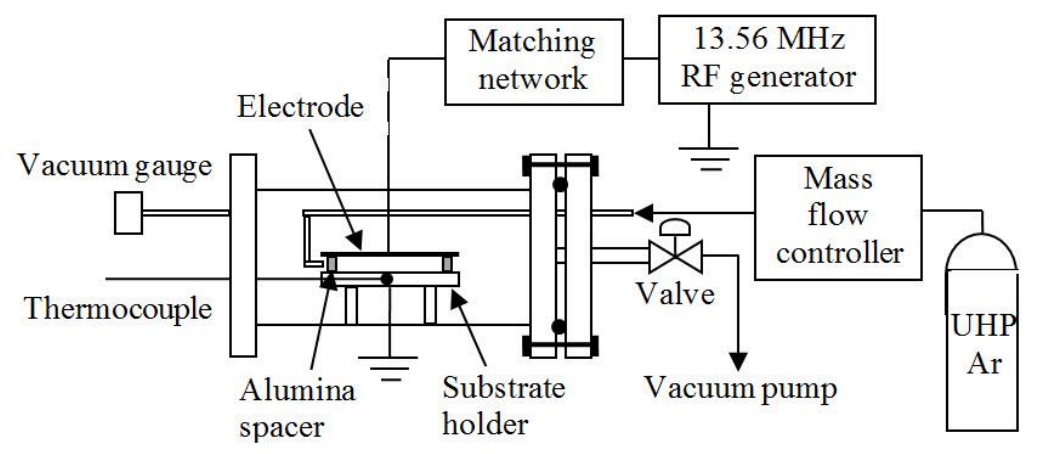

Fig. 1. Schematic diagram of the experimental setup.

\subsection{Silk Specimens}

The specimens for each run had a rectangular size of approximately $5 \times 10 \mathrm{~cm}$, cut from a large piece of woven Thai silk fabric purchased from Huay Kian Silk Fabric Weaver, Moo 10, Chiang Rai province, Thailand. The fabric had not been colored or treated in any way; it was made from $100 \%$ natural Thai silk. Prior to placing the specimen on the specimen holder, it was thoroughly cleaned with methyl alcohol several times to remove any oil attaching to the surface. Afterwards, it was placed in a forced-convection oven at $50^{\circ} \mathrm{C}$ for 20 minutes to achieve complete dryness.

\subsection{Optimized RF Power}

An optimized RF power yielding the highest degree of chitosan grafting was determined. For each run, 2 cleaned and dried specimens were placed on the specimen holder. The plasma treatment was performed under 0.8 Torr of Ar pressure. The RF power was set from $10-180$ watts with the treatment time of 5 minutes.

After plasma treatment, the specimens were quickly submerged and stirred in a $1 \%(\mathrm{w} / \mathrm{v})$ chitosan solution at room temperature for 2 minutes. The solution was prepared by dissolving $1 \mathrm{wt} \%$ of chitosan into a $2 \%(\mathrm{v} / \mathrm{v})$ acetic acid solution, which was adjusted to a $\mathrm{pH}$ of 5.6 by addition of $\mathrm{NaOH}$. The foodgrade chitosan was purchased from Bonafides Marketing Company, Thailand, with the deacetylation degree of $95 \%$ minimum and the molecular weight in the range of $500-1,000 \mathrm{kDa}$. Afterwards, the specimens were washed with deionized water several times to ensure that non-grafted chitosan was completely removed. Specimens were placed in a forced-convection oven at $50^{\circ} \mathrm{C}$ to achieve complete dryness.

Another set of specimens was also directly immersed into the chitosan solution without prior RF plasma activation of the surface. This was done to demonstrate that plasma activation resulted in a higher degree of grafting, which was calculated according to Eq. (1):

$$
\text { Degree of grafting }(\%)=\frac{\mathrm{m}_{1}-\mathrm{m}_{0}}{\mathrm{~m}_{0}} \times 100
$$

where $\quad \mathrm{m}_{1}=$ Mass of silk after chitosan submersion; $\mathrm{m}_{0}=$ Mass of silk before chitosan submersion.

\subsection{Optimized Molecular Weight of Chitosan}

Concentration and molecular weight of chitosan can influence antimicrobial effect on E. coli and S. aureus, especially at low concentration of less than $1 \%$ [18]. However, at $1 \%$ concentration, the value intended to use throughout the present study, molecular weight appeared to have no effect on the properties [18]. Nonetheless, molecular weight of chitosan can affect degree of grafting. Thus, the effect of chitosan molecular weight on the degree of grafting was investigated.

As-received chitosan in the solid state was packed in polystyrene bags and was irradiated with gamma ray from Co-60 at the absorbed dose of 10, 30, 50, 70 and $90 \mathrm{kGy}$ with the absorbed dose rate of 5.63 $\mathrm{kGy} / \mathrm{h}$ (measured on March 2012). As high-energy gamma ray from Co-60 breaks down the molecular chains of chitosan, more absorbed dose resulted in shorter molecular chains and lower molecular weight. 
Irradiation was performed at the Office of Atoms for Peace, Bangkok, and the temperature in the irradiation chamber was approximately $40^{\circ} \mathrm{C}$. Grafting of chitosan with different molecular weights on silk specimens was performed at $120 \mathrm{~W}$ of RF power and the degree of grafting was calculated according to Eq. (1).

\subsection{Hydrophilicity Test}

The ability of silk specimens to be rapidly soaked with chitosan solution was preferred to reduce the soaking time in the chitosan solution. Each silk specimen was gently placed horizontally on the chitosan solution. Time from first contact with chitosan solution to complete submersion was recorded. Both RFplasma treated and non-RF-plasma treated silk specimens were evaluated to determine the difference in hydrophilicity.

\subsection{Antibacterial Evaluation Following Laundering Durability Test}

Chitosan-grafted silk fabrics would occasionally be washed, resulting in a certain amount of chitosan removal. To simulate washing, the laundering durability test was performed by pouring tap water into a 5liter beaker and mixing this with ordinary detergent at $1 \mathrm{~g} / \mathrm{L}$ concentration. The temperature was maintained at $35-36^{\circ} \mathrm{C}$, and the test was performed for 30 minutes per TIS-121 (3-1975) standard.

After each cycle of laundering durability test, the degree of chitosan remaining on the silk specimen was evaluated based on antibacterial properties. Evaluations were performed for E. coli and S. aureus following the AATCC TM 100-2004 protocol [19]. In summary, the test specimens were sterilized before testing by using the autoclave at $121^{\circ} \mathrm{C}, 15 \mathrm{psi}$ for 15 minutes. $1.0 \pm 0.1 \mathrm{ml}$ of the $S$. aureus inoculum was loaded on the swatches of $4.8 \pm 0.1 \mathrm{~cm}$ in diameter, cut from the test fabrics (treated and untreated). After inoculation, the specimens were incubated at $37 \pm 2^{\circ} \mathrm{C}$ for 24 hours. Then, the bacteria were eluted from the specimen swatches by shaking in known amounts of neutralizing solution. The percentage bacteria reduction can be calculated according to Eq. (2):

$$
\% \text { Reduction }(\mathrm{R})=100(\mathrm{C}-\mathrm{A}) / \mathrm{C} \text {, }
$$

where $(\mathrm{A})=$ the number of bacteria recovered from the inoculated treated test specimen swatches in the jar after 24 hours contact time; $(\mathrm{C})=$ the number of bacteria recovered from the inoculated untreated control swatches in the jar at 0 hour contact time.

\section{Results and Discussion}

\subsection{Optimized RF Power}

Figure 2 shows the degree of grafting vs. RF power. Each data point represented an average value obtained from 2 specimens. The value at 0 watt represented the silk specimen without plasma activation prior to submersion in the chitosan solution. The degree of grafting resulted from 2 effects: generation of active sites on silk fibers and increased surface roughness on silk fibers induced by energetic ion bombardment from the plasma.

From $0-30 \mathrm{~W}$, the degree of grafting was roughly identical, indicating that RF power of 10 and $30 \mathrm{~W}$ was too low as it did not significantly etch the silk surface or generate active sites. This result was in good very agreement with that in [7] where hydrophobicity of Indian muga silk fiber was improved by 13.56 $\mathrm{MHz} \mathrm{RF}$ plasma treatment with Ar gas. In that study, the contact angles at 0, 10, 20 and $30 \mathrm{~W}$ were approximately $98,100,115$ and $92^{\circ}$ for the treatment time of $5 \mathrm{~min}$. Thus, the surface roughness actually decreased progressively from 0 to $20 \mathrm{~W}$, and for $30 \mathrm{~W}$ it increased below the vale at $0 \mathrm{~W}$. Results in Fig. 2 indicate that the degree of grafting dropped from 0 to $10 \mathrm{~W}$, and for $30 \mathrm{~W}$ it increased to above the value at $0 \mathrm{~W}$. Thus, for the range of $0-30 \mathrm{~W}$, the result of the present study was in very good agreement with [7]. However, [7] only studied up to $30 \mathrm{~W}$, so no more comparison can be made for higher wattages.

From $30-120 \mathrm{~W}$, the degree of grafting increased with increasing RF power because of the increasing surface roughness and probably because more active sites on silk surface were generated as well. However, beyond $120 \mathrm{~W}$, the degree of grafting dropped drastically, even to the level below that obtained with the RF 
power of $0-30 \mathrm{~W}$. For RF power more than $120 \mathrm{~W}$, although more active sites may be generated, further surface etching must have rendered the condition of the surface unsuitable for chitosan to graft onto. Thus, it can be concluded that, under the experimental setup, the degree of grafting reached the highest value of approximately $30 \%$ at the RF power of $120 \mathrm{~W}$. Therefore, this RF power was used throughout the study.

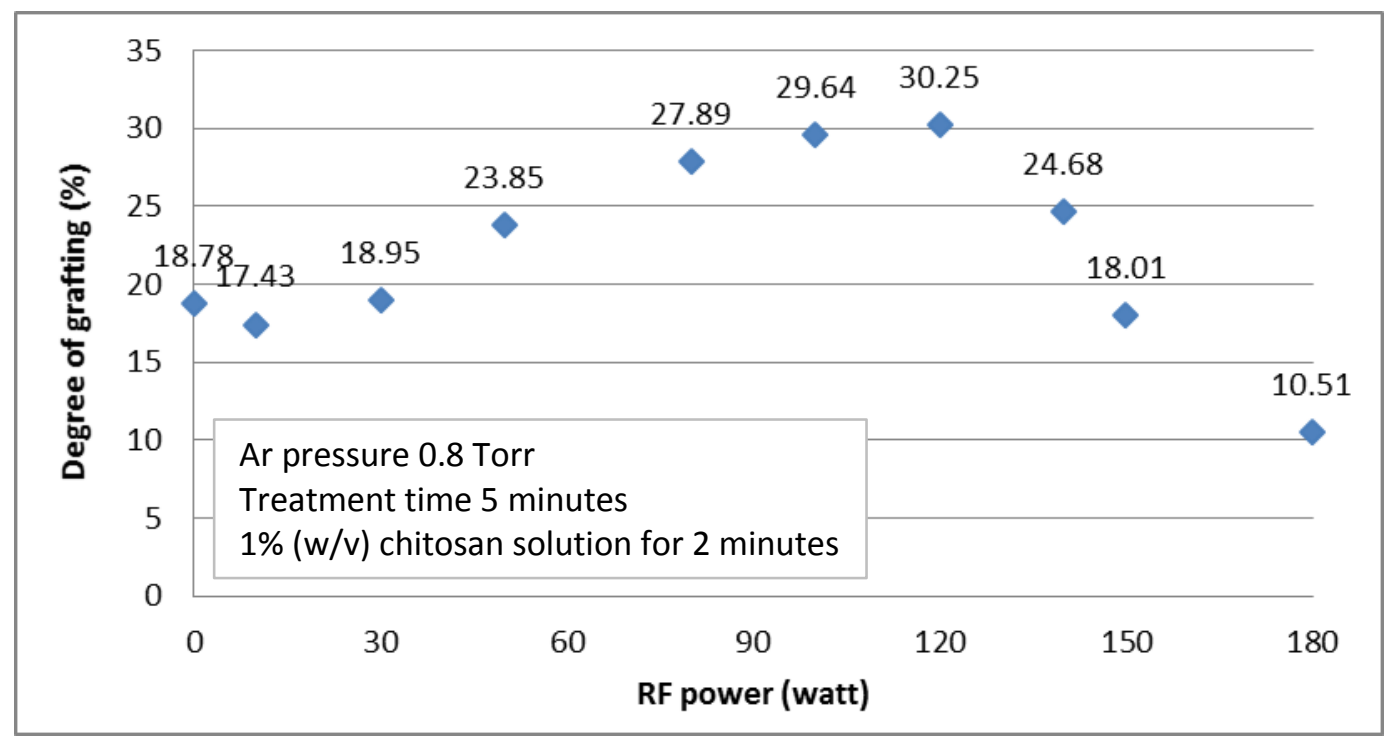

Fig. 2. Degree of grafting vs. RF power.

Figures 3(a) - 3(j) show SEM micrographs of activated silk fibers before chitosan solution submersion.

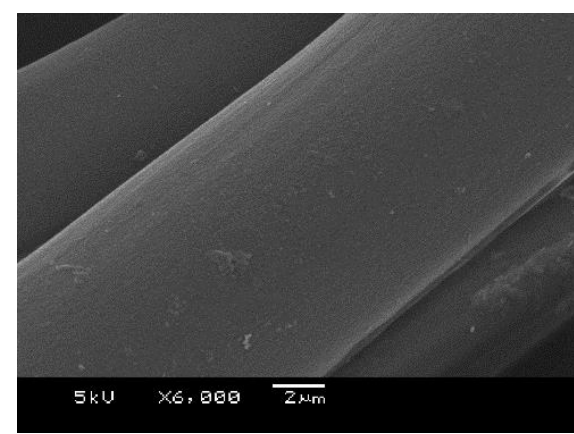

a : $0 \mathrm{~W}$ (untreated)

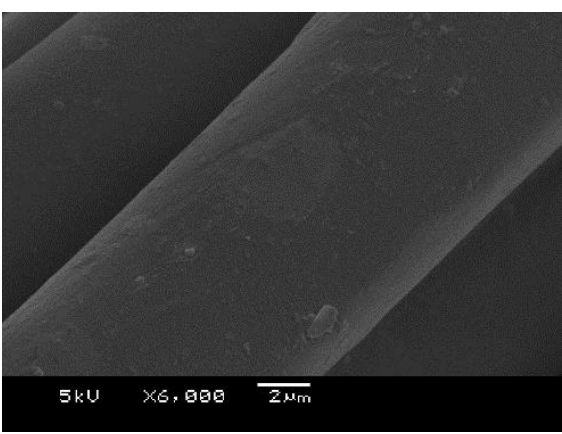

$\mathrm{c}: 30 \mathrm{~W}$

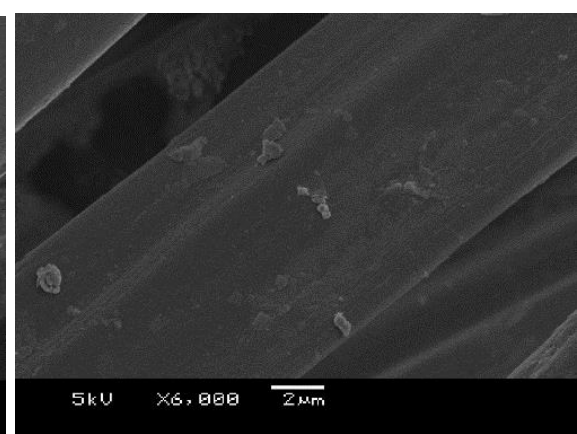

$\mathrm{b}: 10 \mathrm{~W}$

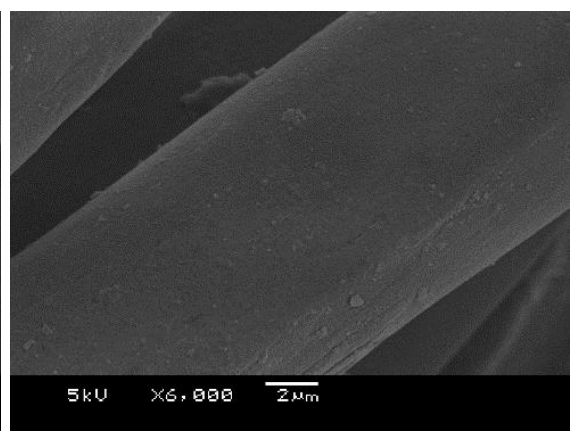

$\mathrm{d}: 50 \mathrm{~W}$ 


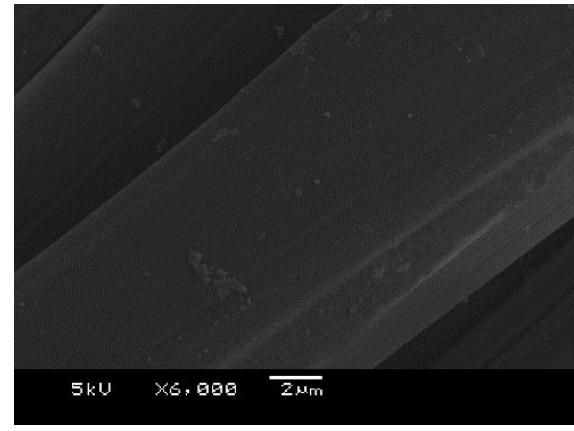

e : $80 \mathrm{~W}$

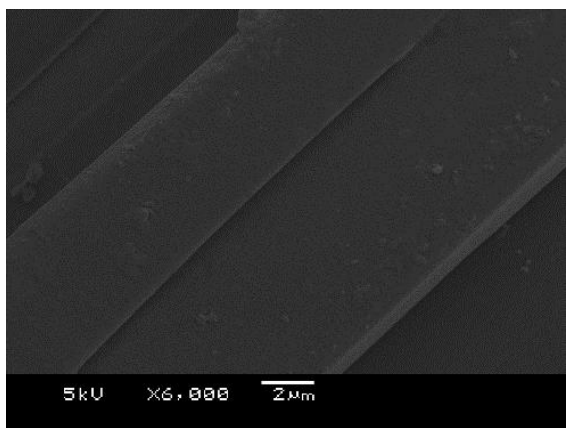

$\mathrm{g}: 120 \mathrm{~W}$

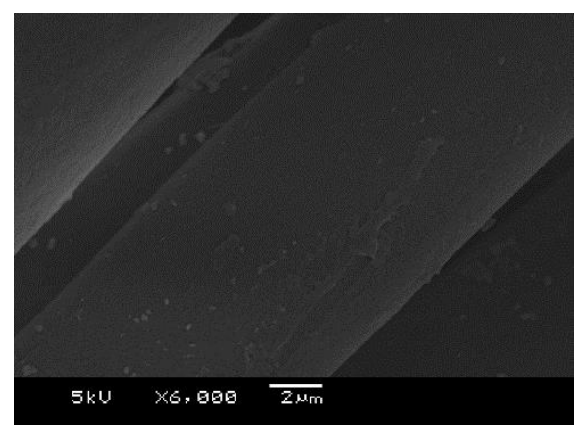

i : $150 \mathrm{~W}$

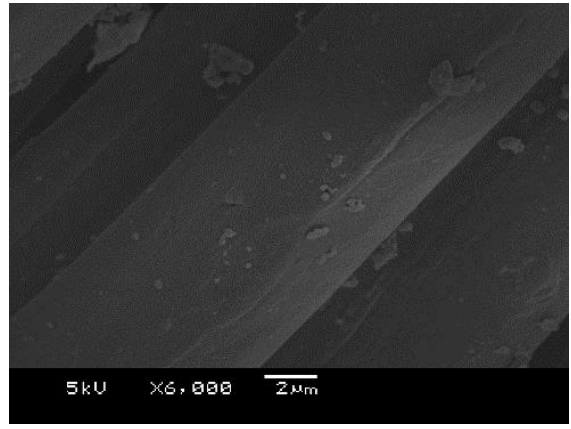

$\mathrm{f}: 100 \mathrm{~W}$

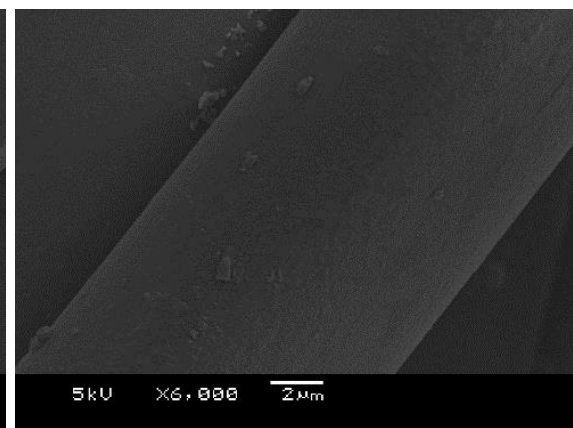

$\mathrm{h}: 140 \mathrm{~W}$

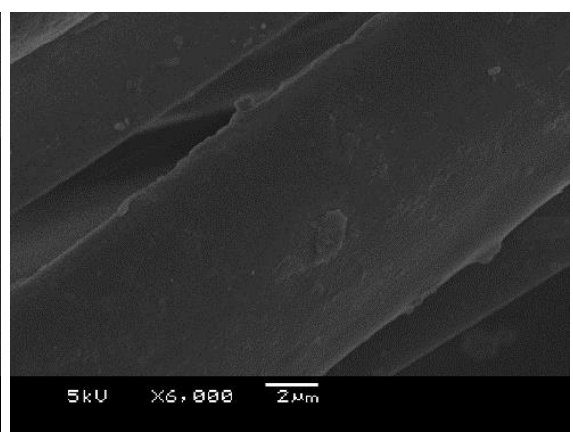

$\mathrm{j}: 180 \mathrm{~W}$

Fig. 3. Scanning electron micrographs of raw silk fibers after RF plasma treatment of various power.

The SEM micrographs reveal several information. The $10 \mathrm{~W}$ plasma treated silk fibers already exhibited more surface roughness than the untreated ones. Increasing the RF power appeared to make fiber surfaces rougher. However, from $120 \mathrm{~W}$ to $140 \mathrm{~W}$, the range of RF power that the grafting percentage started to decrease abruptly, no observation can be made on any obvious alteration on the surface morphology of silk fibers to explain the drop in the degree of grafting, although at $180 \mathrm{~W}$, the fibers appeared to be etched by plasma the most. The underlying mechanism on the abrupt decrease in the grafting percentage is beyond the scope of the present study.

\subsection{Optimized Molecular Weight of Chitosan}

Figure 4 illustrates the effect of chitosan molecular weight (represented by the absorbed dose) on the degree of grafting. 


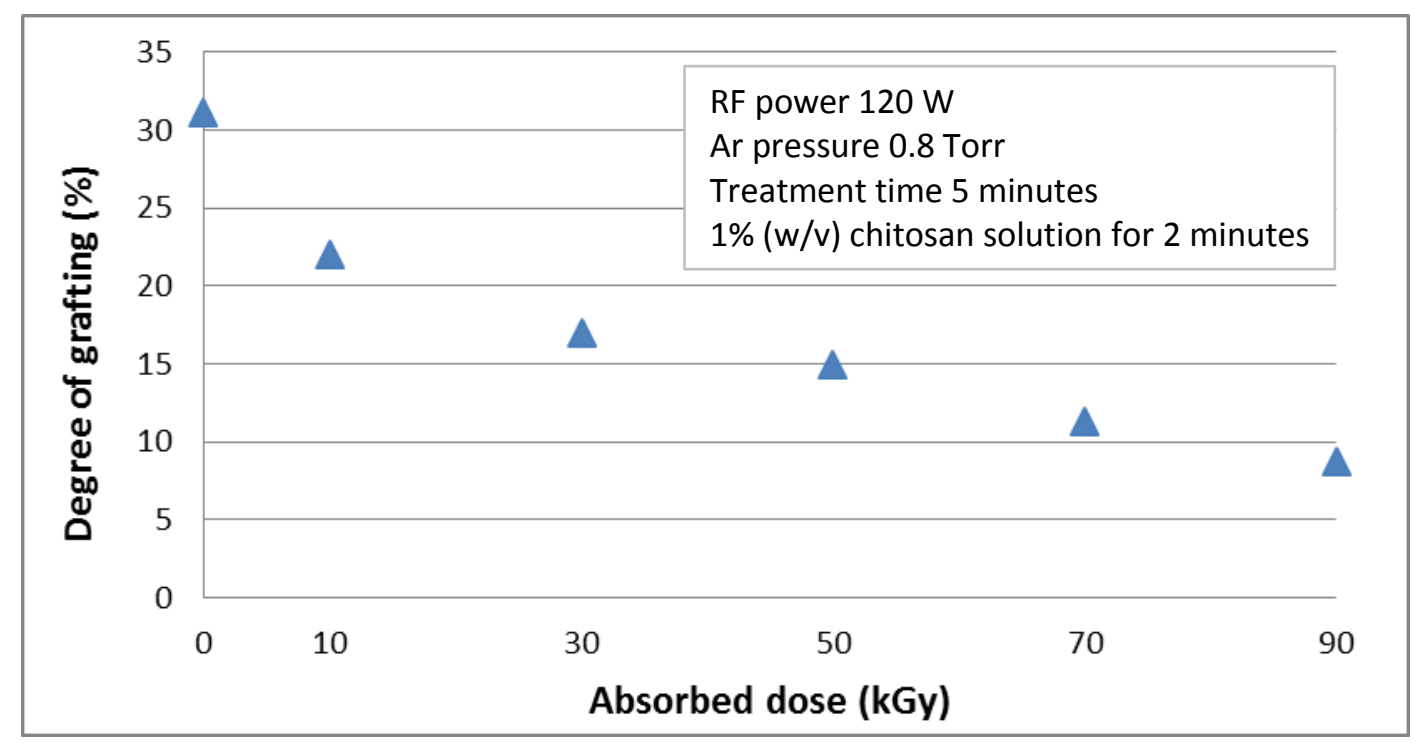

Fig. 4. Effect of chitosan molecular weight on degree of grafting.

A clear effect can be observed. The degree of grafting reduced with increasing absorbed dose (or with reducing molecular weight). This indicates that, with chitosan concentration being identical, a less viscous chitosan can graft more effectively than a more viscous one. From the figure, the unirradiated chitosan yielded the highest degree of grafting. Thus, as-received, unirradiated chitosan at $1 \%(\mathrm{w} / \mathrm{v})$ concentration was used throughout the present study.

\subsection{FTIR and SEM Analysis}

Figure 5 shows the FTIR spectra of raw silk fabrics and silk fabrics grafted with chitosan.

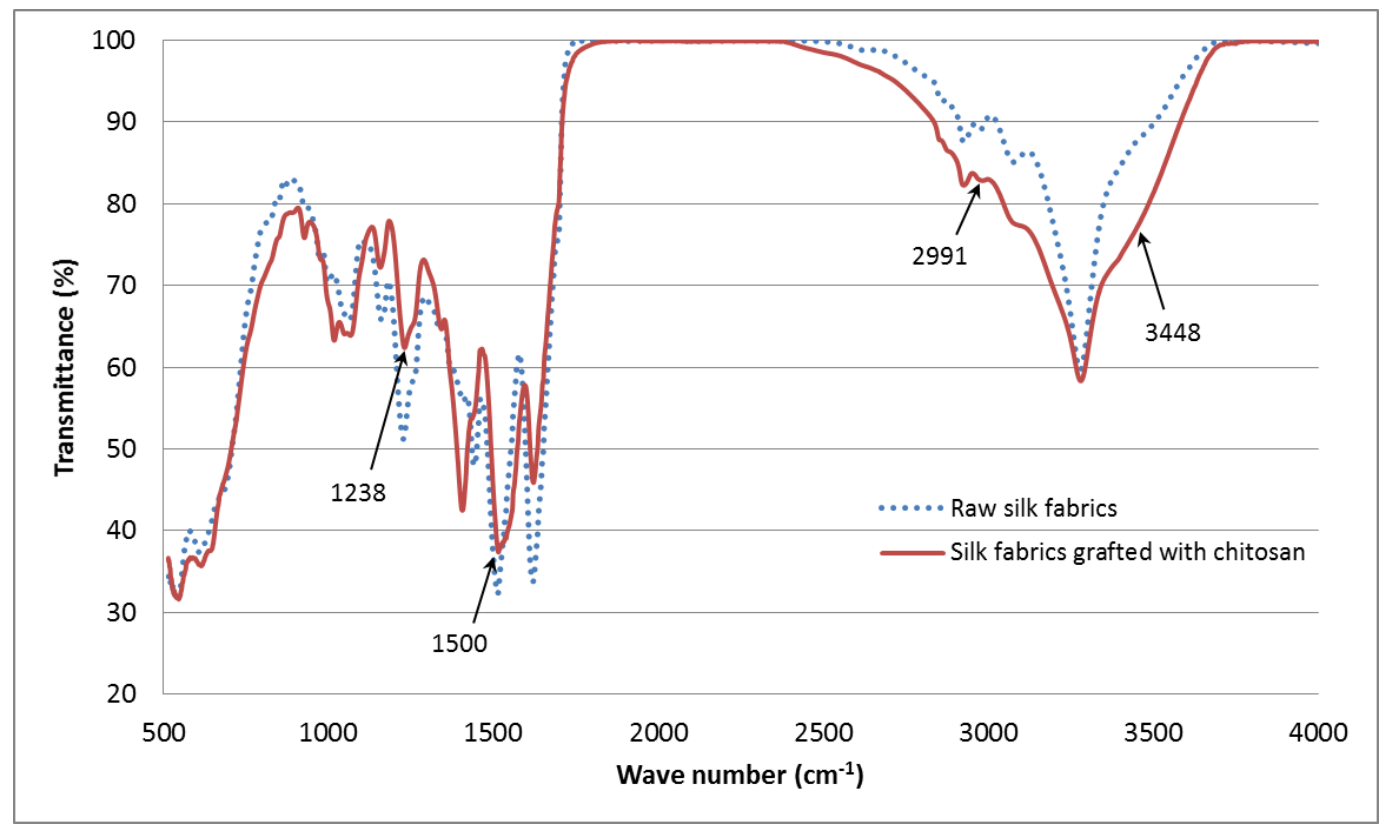

Fig. 5. FTIR spectra of raw silk fabrics and silk fabrics grafted with chitosan.

The following characteristic peaks for chitosan were present, although some were weak: 894 (primary amine), 1070 (carbonyl group), 1238 (aliphatic amines), 1500 ( $1^{\circ}$ amines and amides, indicating the condensation reaction between $-\mathrm{C}=\mathrm{O}$ of silk fibroin and $\mathrm{NH}_{2}$ of chitosan resulting in $-\mathrm{C}-\mathrm{O}-\mathrm{NH}$ with a 
strong covalent bond), 2991 (hydroxyl group) and 3448 (amino group of primary amine or amide). This result confirmed the presence of chitosan on the silk specimen.

Figure 6 displays scanning electron micrographs of raw silk fibers and chitosan-coated silk fibers before and after the laundry process.

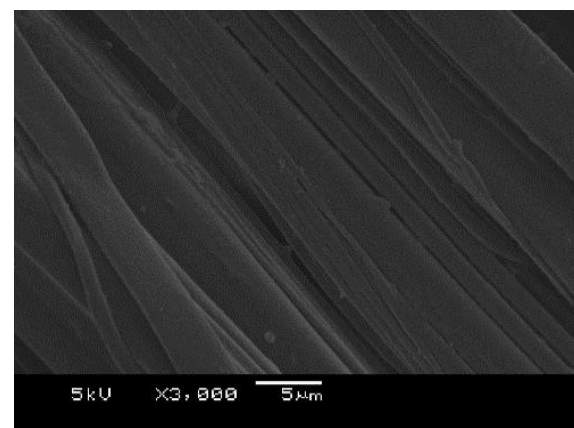

a

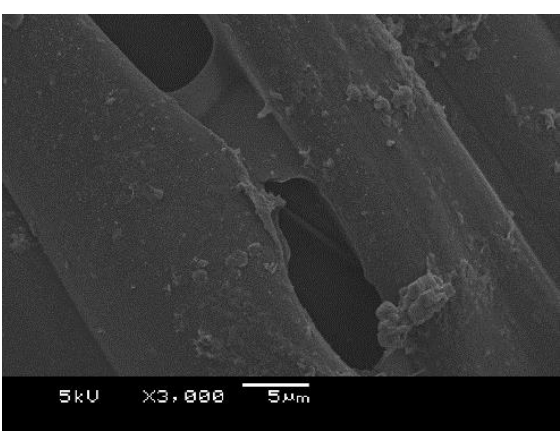

C

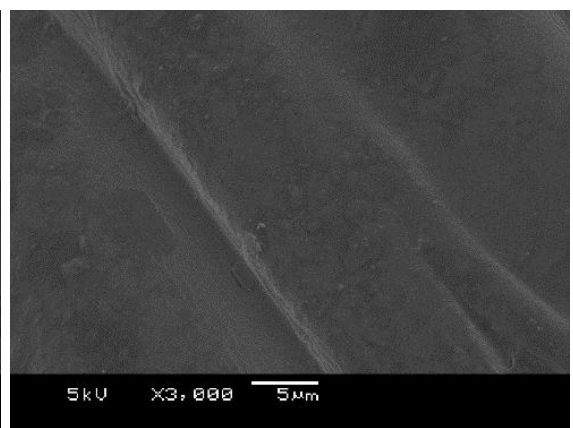

b

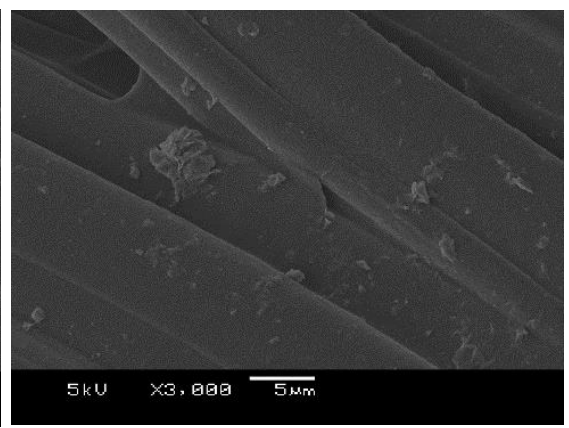

d

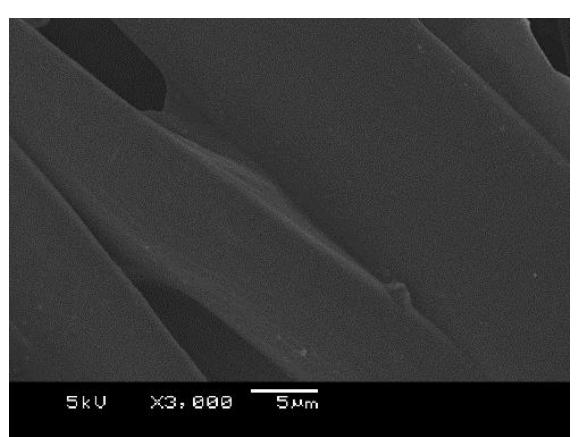

e

Fig. 6. Scanning electron micrographs of raw silk fibers and chitosan-coated silk fibers before and after laundry process: a) before RF plasma activation; b) with RF plasma activation and unwashed; c) washed 1 time; d) washed 2 times; e) washed 5 times

The scanning electron micrographs revealed that silk fibers were coated with chitosan almost everywhere. With increasing number of washing cycles, chitosan appeared to be present less and less. This result was expected since a certain amount of chitosan would be removed during laundry. Chitosan removal would have a direct effect on antibacterial ability discussed Section 3.4.

In addition, for physical appearances under visual observation, after undergoing plasma activation and chitosan coating, all silk specimens exhibited the same white to slight yellow color. Thus, the plasma treatment process and chitosan grafting did not alter the physical appearance of the silk fabrics. 


\subsection{Hydrophilicity Test}

Table 1 displays the result of the hydrophilicity test. Because each silk specimen weighed slightly different, which would result in different gravitational force acting on the specimens, the time to complete submersion was measured in seconds per gram of silk fabric.

Table 1. Result of hydrophilicity test.

\begin{tabular}{ccccccc}
\hline \multirow{2}{*}{$\begin{array}{c}\text { Sample } \\
\text { no. }\end{array}$} & $\begin{array}{c}\text { Fabric mass } \\
\mathbf{( g )}\end{array}$ & $\begin{array}{c}\text { Time to } \\
\text { complete } \\
\text { submersion (s) }\end{array}$ & $\begin{array}{c}\text { Submersion } \\
\text { time per } \\
\text { mass (s/g) }\end{array}$ & $\begin{array}{c}\text { Fabric mass } \\
\mathbf{( g )}\end{array}$ & $\begin{array}{c}\text { Time to } \\
\text { complete } \\
\text { submersion (s) }\end{array}$ & $\begin{array}{c}\text { Submersion } \\
\text { time per mass } \\
(\mathbf{s} / \mathbf{g})\end{array}$ \\
\hline 1 & 0.7845 & 14.2 & 18.1 & 0.7874 & $1,920.7$ & $2,439.3$ \\
2 & 0.7922 & 15.1 & 19.1 & 0.8166 & $2,040.8$ & $2,499.2$ \\
3 & 0.8101 & 18.3 & 22.6 & 0.7700 & $1,933.6$ & $2,511.2$ \\
4 & 0.9229 & 24.9 & 27.0 & 0.7909 & $1,961.5$ & $2,480.1$ \\
\hline & Average & $\mathbf{2 1 . 7}$ & & & $2,482.5$ \\
\hline
\end{tabular}

It was evident from results shown in the table that fabrics with RF plasma treatment could be submerged into (or absorb) the chitosan solution substantially faster than those without the treatment. Thus, RF plasma treatment of $120 \mathrm{~W}$ for 5 mins significantly increased the hydrophilicity of silk fabrics. This effect was due to additional surface roughness on silk fibers induced by energetic ion bombardments from the plasma as can be observed in SEM micrographs in Fig. 3. Another benefit of increased hydrophilicity is the ability of the treated fabrics to allow human perspiration to flow through more effectively, providing added comfort in tropical-zone countries where weather is hot and humid.

\subsection{Antibacterial Evaluation}

Table 2 summarizes results of antibacterial evaluations. Figures $7 \mathrm{a}$ and $7 \mathrm{~b}$ show the bacterial growth during the antibacterial evaluation.

Table 2. Antibacterial evaluation for E. coli and S. aureus.

\begin{tabular}{lccc}
\hline Treatment & \% $\boldsymbol{E}$. coli reduction (1) & $\mathbf{\%} \boldsymbol{S}$. aureus reduction (2) & Ratio of (2) to (1) \\
\hline NP, NC, & 0 & 0 & - \\
NP, NC, 1 & 0.79 & 2.60 & 3.29 \\
NP, NC, 2 & 1.18 & 1.30 & 1.10 \\
NP, NC, 3 & 0.59 & 3.25 & 5.51 \\
NP, NC, 4 & 0.98 & 8.44 & 8.61 \\
NP, NC, 5 & 1.38 & 12.34 & 8.94 \\
NP, C, 0 & 100 & 100 & 1.00 \\
NP, C, 1 & 97.24 & 98.05 & 1.01 \\
NP, C, & 98.03 & 97.40 & 0.99 \\
NP, C, 3 & 94.49 & 94.81 & 1.00 \\
NP, C, 4 & 91.14 & 90.91 & 1.00 \\
NP, C, 5 & 88.78 & 90.26 & 1.02 \\
P, C, 0 & 99.80 & 100 & 1.00 \\
P, C, 1 & 100 & 99.35 & 0.99 \\
P, C, 2 & 98.82 & 98.70 & 1.00 \\
P, C, 3 & 98.23 & 98.05 & 1.00 \\
P, C, 4 & 99.21 & 96.75 & 0.98 \\
P, C, 5 & 95.87 & 94.16 & 0.98 \\
\hline
\end{tabular}

$N P=$ No RF plasma activation

$P=$ With RF plasma activation

$N C=$ No cbitosan solution submersion

$C=$ With chitosan solution submersion

$0,1,2,3,4,5=$ Number of washing cycles $(0=$ not washed $)$ 


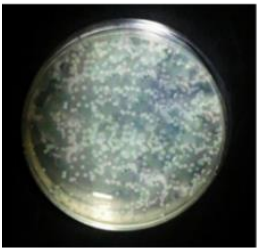

NP, NC, 0

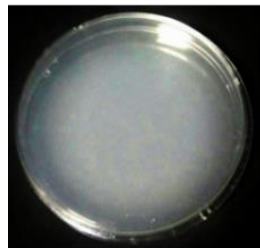

NP, C, 0

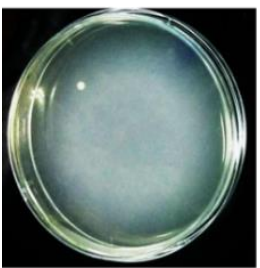

P, C, 0

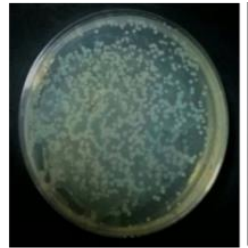

NP, NC, 1

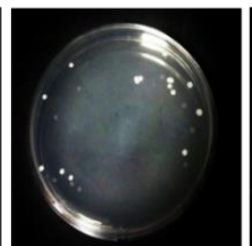

NP, C, 1

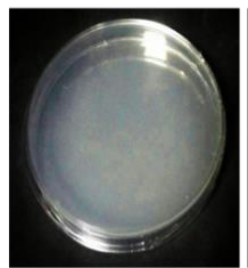

P, C, 1

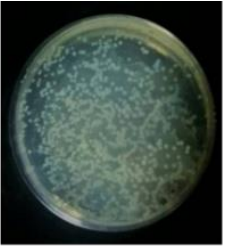

NP, NC, 2

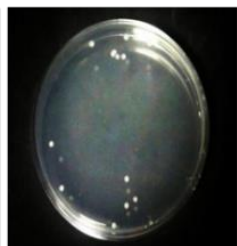

NP, C, 2

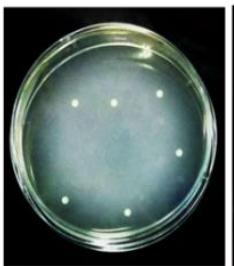

P, C, 2

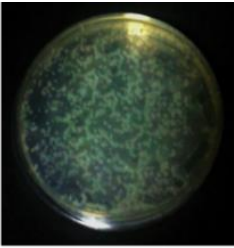

NP, NC, 3

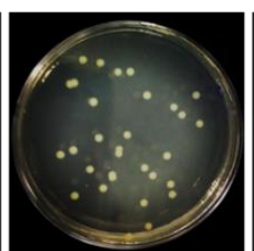

NP, C, 3

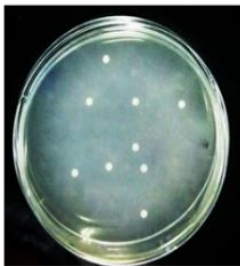

P, C, 3

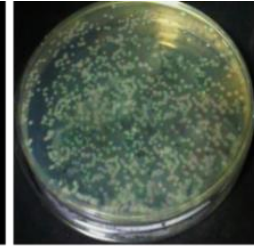

NP, NC, 4

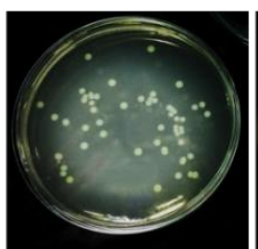

NP, C, 4

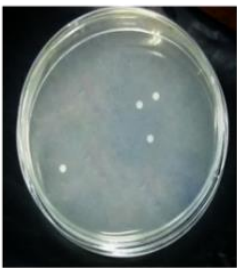

P, C, 4

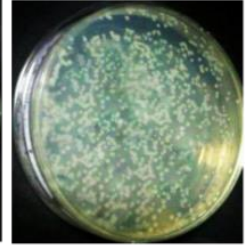

NP, NC, 5

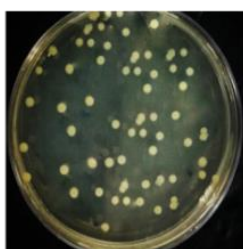

NP, C, 5

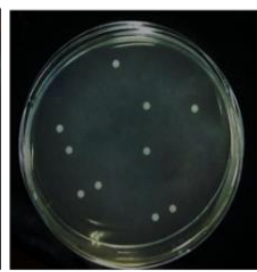

P, C, 5

a. E. Coli

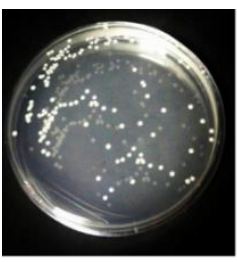

NP, NC, 0

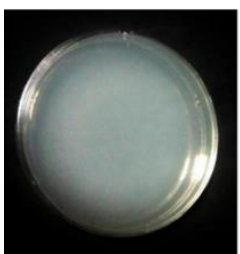

NP, C, 0

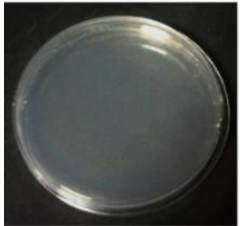

P, C, 0

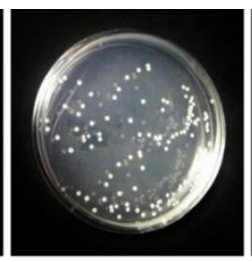

NP, NC, 1

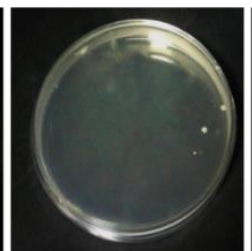

NP, C, 1

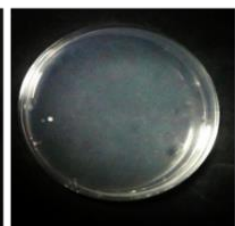

P, C, 1

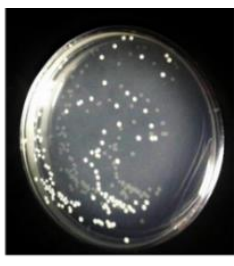

NP, NC, 2

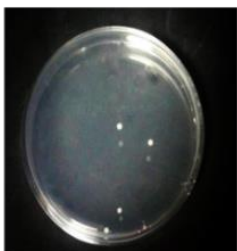

NP, C, 2

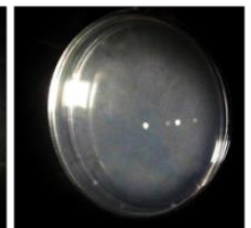

P, C, 2

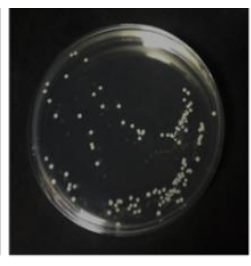

NP, NC, 3

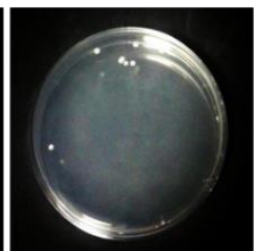

NP, C, 3

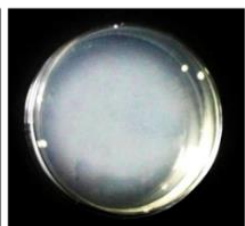

P, C, 3

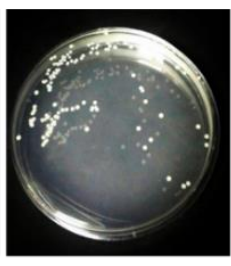

NP, NC, 4

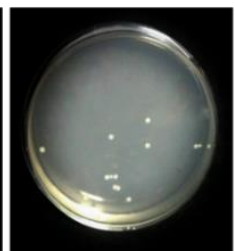

NP, C, 4

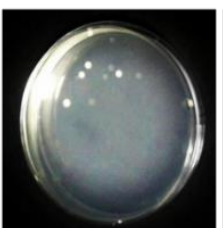

P, C, 4

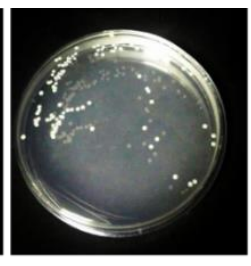

NP, NC, 5

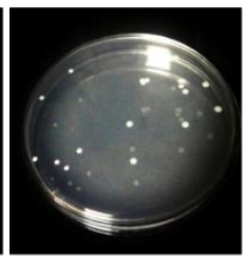

NP, C, 5

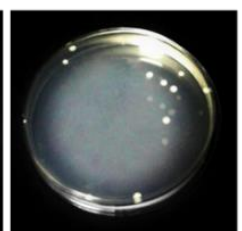

P, C, 5

$N P=N o$ RF plasma activation

\section{b. S. Aureus}

$P=$ With RF plasma activation

$N C=$ No chitosan solution submersion

$C=$ With chitosan solution submersion

$0,1,2,3,4,5=$ Number of washing cycles $(0=$ not washed $)$

Fig. 7. Bacterial growth during the antibacterial evaluation. 
Results reveal that for both evaluations, silk specimens without RF plasma treatment (NP) and without chitosan coating (NC) offer no antibacterial ability, as expected. However, it was of interest to note that with an increasing number of washing cycles, the antibacterial ability increased slightly. The exact mechanism that helped increase the antibacterial ability is beyond the scope of this study. It may be because detergent in newly-washed fabrics provide a short-term antibacterial effect. 2 - 3 continuous rounds of washing without wearing may accumulate this short-term detergent accumulation effect. However, the increase was insignificant compared to specimens coated with chitosan.

According to the ratio shown in the last column in Table 2, it was also observed that for silk specimens without RF plasma treatment (NP) and without chitosan coating (NC), after having washed at least 1 time, the antibacterial activity to $S$. aureus was much higher than to E. coli.

For silk specimens without RF plasma treatment (NP) but with chitosan coating (C), without any washing (0), they offered $100 \%$ protection against both E. coli and S. aureus. This result was expected because essentially all of the coated chitosan was still present on the fabrics. However, for the overall trend, the antibacterial ability reduced with increased washing cycles, and it dropped to approximately $90 \%$ after 5 washing cycles.

Without RF plasma treatment to generate active sites on silk fabric, chitosan was not expected to form strong covalent bonds on the fabric surface. All or most of chitosan was expected to be washed away after a few washing cycles. However, the result indicates otherwise. Chitosan must have somehow formed covalent bonds on the silk surface, but exact mechanism of bond formation is beyond the scope of the present study. Therefore, even without RF plasma treatment prior to chitosan solution submersion, a substantial quantity of chitosan will still be present on silk fabric following a few washing cycles.

For silk specimens with RF plasma treatment $(\mathrm{P})$ and chitosan coating $(\mathrm{C})$, without any washing $(0)$, they offered $100 \%$ protection against both bacteria species. This result was expected because essentially all of the coated chitosan was still present on the fabrics. However, for the overall trend, the antibacterial ability reduced with increased washing cycles, and it dropped to approximately $95 \%$ after 5 washing cycles. This removal was expected, as weakly-bonded chitosan was washed away and less chitosan was present. This performance was slightly better than that of silk specimens without RF plasma treatment (NP) but with chitosan coating (C).

Similar to findings in [20] where, at 1\% chitosan concentration and high molecular weight (such as 305 $\mathrm{kDa}$ ), the antimicrobial activities to both E. coli and S. aureus was identical at $100 \%$, chitosan-coated silk specimens (both NP and P) in the present study before washing offered essentially $100 \%$ protection as well. After several rounds of washing, the degree of antimicrobial protection for both bacteria were roughly the same, indicated by the ratio shown in the last column in Table 2 which are close to unity for all washing cycles.

Although the benefit of RF plasma treatment on chitosan retainment may appear to be only slightly better; if the fabrics would be washed many more times, the significance of chitosan retainment on the surface would become clearer as shown in Fig. 8 .

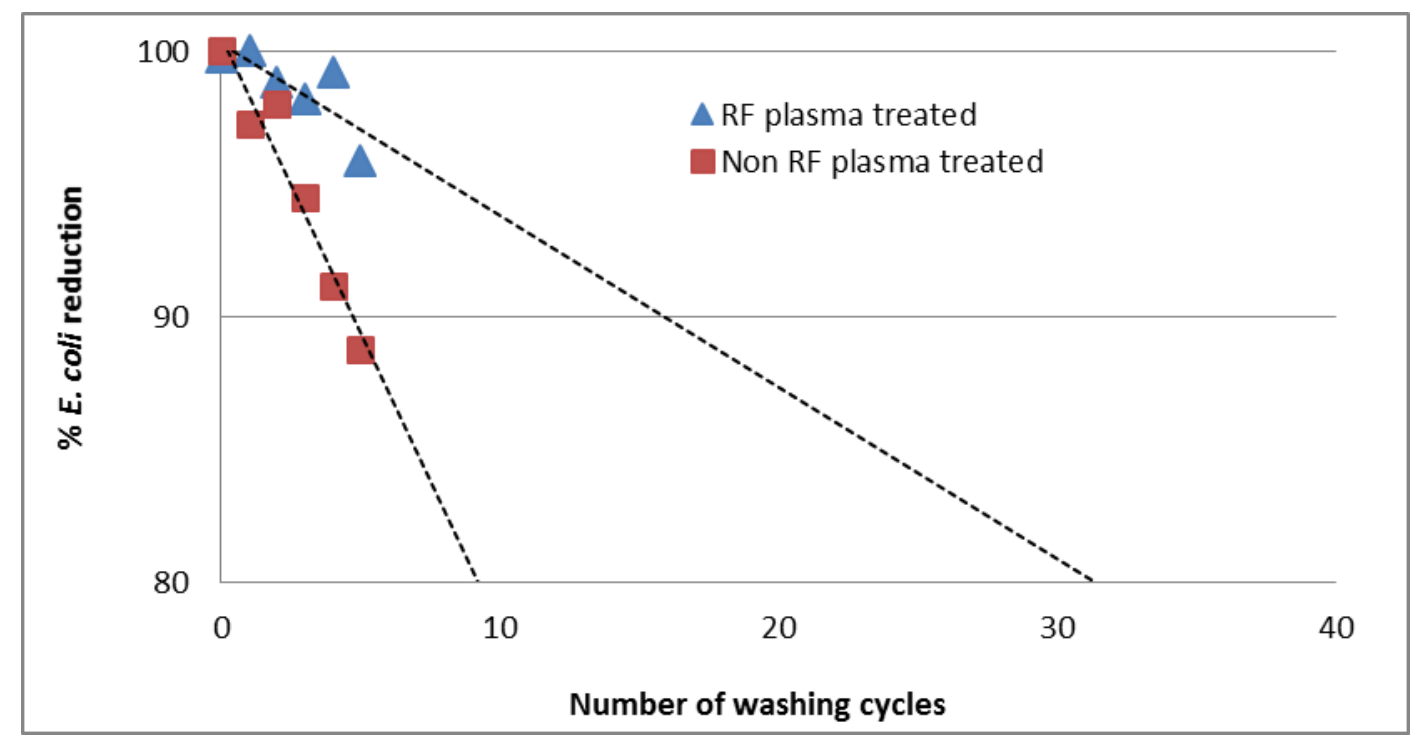

a. E. coli 


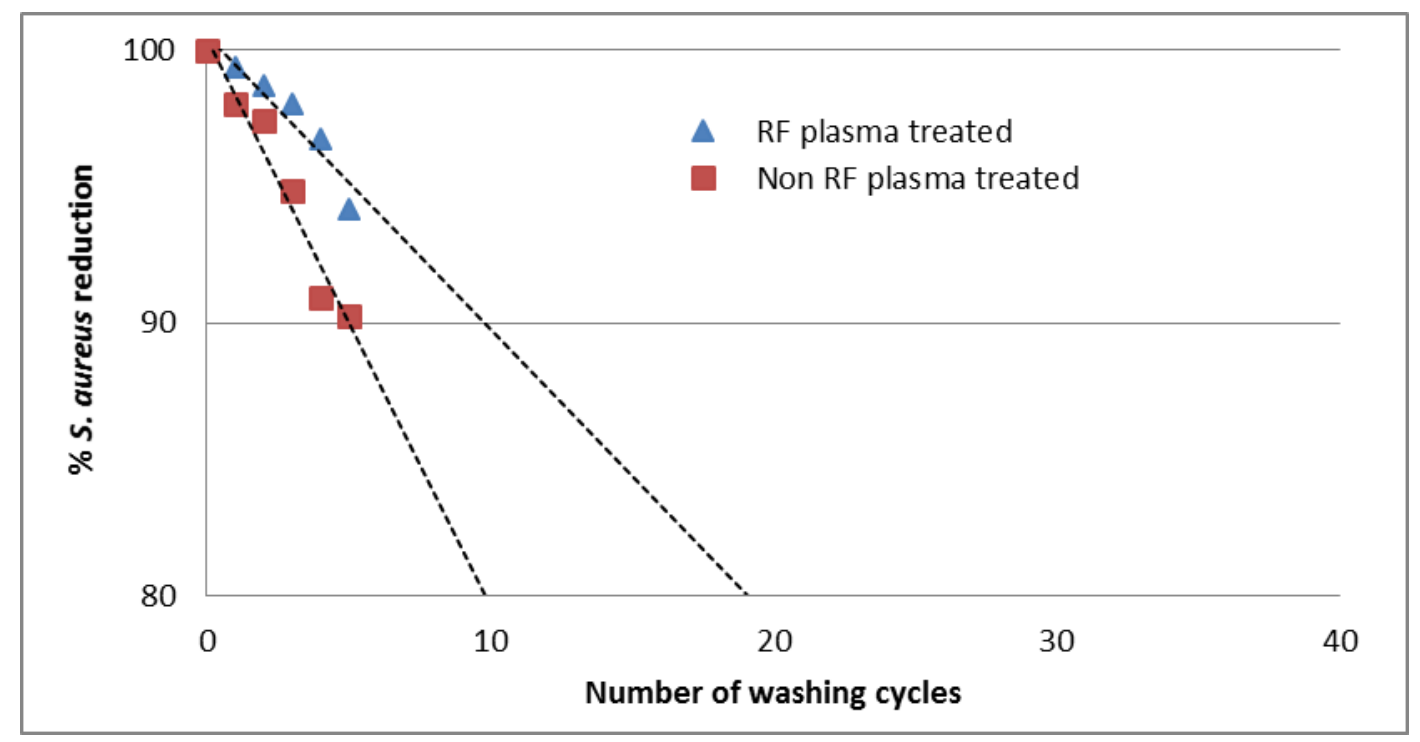

b. S. aureus

Fig. 8. Forward prediction following a linear trend line for antibacterial ability against a) E. coli and b) $S$. aureus.

Extrapolation of linear trend lines for the RF plasma treated specimens reveals that for the case of E. coli, it would take approximately 16 washing cycles to reduce the antibacterial ability to $90 \%$, assuming that the linear trend continues beyond 5 washing cycles. For the case of S. aureus, it would take approximately 10 washing cycles to reduce the antibacterial ability to $90 \%$, assuming that the linear trend continues beyond 5 washing cycles. Fabrics without RF plasma treatment will have their antibacterial ability for E. coli and S. aureus reduced to $90 \%$ after only about 5 washing cycles. These predictions, relying upon extrapolation of linear trend lines, indicate that plasma activation of silk fabrics should be performed before chitosan coating to ensure durable retainment of chitosan on the fabrics following numerous washing cycles.

\section{Conclusions}

This experimental work successfully utilized $13.56 \mathrm{MHz}$ RF plasma treatment to durably coat Thai silk fabrics with chitosan for antibacterial property. Uncolored and untreated Thai silk fabrics were activated in Ar plasma for 5 minutes with an optimized RF power of $120 \mathrm{~W}$ under the Ar pressure of 0.8 Torr. After plasma activation, specimens were submerged and stirred in a $1 \%(\mathrm{w} / \mathrm{v})$ chitosan solution. FTIR analysis confirmed the presence of chitosan on the silk fabrics. From SEM analysis, chitosan was observed to coat silk fibers almost everywhere. RF plasma treated fabrics were able to absorb the chitosan solution substantially faster than those without the treatment. Another benefit of increased hydrophilicity is the ability of the treated fabrics to allow human perspiration to flow through more effectively, providing more wearing comfort in tropical-zone countries.

Antibacterial properties against E. coli and $S$. aureus reduced with increased washing cycles, and it dropped to approximately $95 \%$ after 5 rounds of washing. Extrapolation of linear trend lines revealed that for the case of E. coli, it will take approximately 16 washing cycles to reduce the antibacterial ability to $90 \%$. For the case of $S$. aureus, it will take approximately 10 washing cycles to reduce the antibacterial ability to $90 \%$. Fabrics without RF plasma treatment prior to chitosan solution submersion will have their antibacterial ability for E. coli and S. aureus reduced to $90 \%$ after only about 5 washing cycles. The findings of this research offer a new and highly environmentally-friendly coating technique of silk with chitosan for potential uses in the textile industry to increase the commercial value of silk, because the only chemical required is the environmentally-friendly chitosan solution. 


\section{Acknowledgements}

The authors would like to thank the Asahi Glass Foundation, Chulalongkorn University Centenary Academic Development Project for the financial support for equipment and materials. The authors appreciate Asst. Prof. Suvit Punnachaiya for his advice on the RF power supply system. The authors wish to express our appreciation to Assoc. Prof. Chayagrit Siri-Upathum for his advice on chitosan. The authors wish to thank Prof. Suttichai Assabumrungrat and Assoc. Prof. Dr. Siriporn Damrongsakkul for their advices on the manuscript.

\section{References}

[1] F. F. Shi, "Recent advances in polymer thin films prepared by plasma polymerization synthesis, structural characterization, properties and applications," Surf Coat Tech, vol. 82, no. 1-2, pp. 1-15, Jul. 1996.

[2] Y. Wang, J. Zhang, and X. Shen, "Surface structures tailoring of hexamethyldisiloxane films by pulse rf plasma polymerization," Mater Chem Phys, vol. 96, no. 2-3, pp. 498-505, Apr. 2006.

[3] P. Chaivan, N. Pasaja, D. Boonyawan, P. Suanpoot, and T. Vilaithong, "Low-temperature plasma treatment for hydrophobicity improvement of silk," Surf Coat Tech, vol. 193, no. 1-3, pp. 356-360, Apr. 2005.

[4] P. Suanpoot, K. Kueseng, S. Ortmann, R. Kaufmann, C. Umongno, P. Nimmanpipug, D. Boonyawan, and T. Vilaithong, "Surface analysis of hydrophobicity of Thai silk treated by $\mathrm{SF}_{6}$ plasma," Surf Coat Tech, vol. 202, no. 22-23, pp. 5543-5549, Aug. 2008.

[5] S. Li and D. Jinjin, "Improvement of hydrophobic properties of silk and cotton by hexafluoropropene plasma treatment," Appl Surf Sci, vol. 253, no. 11, pp. 5051-5055, Mar. 2007.

[6] K. Kamlangkla, S. K. Hodak, and J. Levalois-Grützmacher, "Multifunctional silk fabrics by means of the plasma induced graft polymerization (PIGP) process," Surf Coat Tech, vol. 205, no. 13-14, pp. 3755-3762, Mar. 2011.

[7] D. Gogoi, A. J. Choudhury, J. Chutia, A. R. Pal, N. N. Dass, D. Devi, and D. S. Patil, "Enhancement of hydrophobicity and tensile strength of muga silk fiber by radiofrequency Ar plasma discharge," Appl Surf Sci, vol. 258, no. 1, pp. 126-135, Oct. 2011.

[8] S. Davarpanah, N. M. Mahmoodi, M. Arami, H. Bahrami, and F. Mazaheri, "Environmentally friendly surface modification of silk fiber: Chitosan grafting and dyeing," Appl Surf Sci, vol. 255, no. 7, pp. 4171-4176, Jan. 2009.

[9] M. Ranjbar-Mohammadi, M. Arami, H. Bahrami, F. Mazaheri, and N. M. Mahmoodi, "Grafting of chitosan as a biopolymer onto wool fabric using anhydride bridge and its antibacterial property," Colloid Surface B, vol. 76, no. 2, pp. 397-403, Apr. 2010.

[10] D. Wongsawaeng, C. Jumpee, and V. Ratnitsai, "Inhibition of aspergillus on peanuts by coating with chitosan," Chulalongkorn Univ., Bangkok, Thailand, 2013.

[11] D. Wongsawaeng, C. Jumpee, and V. Ratnitsai, "Prolonging shelf life of brown rice by coating with chitosan," Chulalongkorn Univ., Bangkok, Thailand, 2014.

[12] S. Yaset, "Prolonging shelf life of hen eggs by coating with chitosan prepared from gamma irradiation," M.S. thesis, Department of Nuclear Technology, Faculty of Engineering, Chulalongkorn Univ., Bangkok, Thailand, 2011.

[13] Y. Siripholbun, "Edible coating films for prolonging shelf-life of Durian Pulp CV. Monthong," M.S. thesis, Department of Chemical Engineering, Faculty of Engineering, Chulalongkorn Univ., Bangkok, Thailand, 2005.

[14] N. Charoenruay, "Effect of calcium chloride and chitosan on postharvest shelf life and quality of Okra pods Abelmoschus esculentus (L.) Moench," M.S. thesis, Botany. Dept., Chulalongkorn Univ., Bangkok, Thailand, 2005.

[15] J. Yueming, J. Li, and W. Jiang, "Effect of chitosan coating on shelf life of cold-store litchi fruit at ambient temperature," LWT-Food Sci Technol, vol. 38, no. 7, pp. 757-761, Nov. 2005.

[16] D. D. Shao, J. Hu, X. K. Wang, and M. Nagatsu, "Plasma induced grafting multiwall carbon nanotubes with chitosan for 4,4'-dichlorobiphenyl removal from aqueous solution," Chem EngJ, vol. 170, no. 2-3, pp. 498-504, Jun. 2011. 
[17] H.-J. Tseng, S.-H. Hsu, M.-W. Wu, T.-H. Hsueh, and P.-C. Tu, "Nylon textiles grafted with chitosan by open air plasma and their antimicrobial effect," Fiber Polym, vol. 10, no. 1, pp. 53-59, Feb. 2009.

[18] Y. Seki, M. Sarikanat, K. Sever, S. Erden, and H. A. Gulec, "Effect of the low and radio frequency oxygen plasma treatment of jute fiber on mechanical properties of jute fiber/polyester composite," Fiber Polym, vol. 11, pp. 1159-1164, 2010.

[19] AATCC Test Method 100-2004, Antibacterial activity assessment of textile materials: Percentage reduction method, AATCC technical manual, pp. 149-150, 2004.

[20] L.-Y. Zheng and J.-F. Zhu, "Study on antimicrobial activity of chitosan with different molecular weights," Carbobydr. Polym., vol. 54, pp. 527-530, 2003. 